\title{
Estimation of Heat Stress Tolerance for Yield and its Contributing Attributes in Bread Wheat
}

\author{
Pawan Kumar $^{1 *}$, Hoshiyar Singh ${ }^{1}$, Jhabar Singh ${ }^{2}$ and R.N. Choudhary ${ }^{3}$ \\ ${ }^{1}$ Division of Plant Breeding and Genetics, ${ }^{2}$ Division of Agriculture Statistics, ${ }^{3} G \& P B$ \\ Commissionerate College of Education, Jaipur, Rajasthan, India \\ Rajasthan Agricultural Research Institute, Durgapura, Jaipur-302018, India \\ *Corresponding author
}

\section{A B S T R A C T}

\begin{tabular}{|l|}
\hline Ke y w o r d s \\
Wheat, Heat \\
susceptibility index, \\
Thermal stress \\
tolerant
\end{tabular}

In bread wheat (Triticum aestivum L. em. Thell.), development of thermal stress tolerant cultivars and realization of potential at yield in hot climate is enshrined goal of our breeding program. By keeping the above fact in view, the present study was conducted in two environments i.e. normal and late sown condition with 10 diverse genotypes, their 45 $\mathrm{F}_{1}$ 's and $\mathrm{F}_{2}$ 's to magnify the yield level of wheat in high temperature areas by selecting stress tolerant parents and cross combinations. In the present study, as per the HSI value of parents and crosses genotypes were classified in to four different categories i.e. highly heat tolerant (HSI < 0.50), heat tolerant (HSI: 0.51-0.75), moderately heat tolerant (HSI: 0.76 1.00) and heat susceptible (HSI > 1.00). An overall assessment of parents for all the studied characters indicated that parent PBW 343, Raj 4079, Raj 4083 and PBW 590590 were found to be desirable for most of the characters on the basis of HIS while among the crosses, PBW 343 x Raj 4079, PBW 396 x Raj 4120,PBW 502 x PBW 590, C 306 x DBW 88 and PBW 502 x Raj 4079 displayed maximum tolerance as they attained top three rank for more than two characters.

\section{Introduction}

Wheat (Triticum aestivum L. em. Thell.) is the most important agricultural product of the globe and among the major three cereal crops that provides 20 per cent of the total energy requirement in human food (Shewry, 2009). Approximately $90 \%$ of global production of wheat is consumed directly by humans and consequently wheat supplies 20 per cent of the world total plant derived edible dry matter (Evans, 1993). World production of wheat in 2014-15 was 734.1 million tonnes, making it the third most-produced cereal after maize and rice (Anonymous, 2016).

Our country has witnessed spectacular growth in production and productivity of wheat which has contributed to make the country selfsufficient with respect to food grains production. The area and production of wheat in India during year 2016-17 was recorded 30.72 million ha and 97.44 million tonnes with an average productivity of $3172 \mathrm{~kg}$ ha-1 (Anonymous, 2017). 
In Rajasthan, area and production of wheat during 2016-17 was recorded as 3.19 million ha and 11.00 million tonnes, respectively with an average productivity of $3368 \mathrm{~kg}$ ha-1 (anonymous, 2017). Yield potential of any variety is the result of interaction between genotypes and environment. In the present scenario, climatic conditions are changing due to the effect of global warming. During the post anthesis stages, increased temperature during grain development (in late February and March) terminates grain growth prematurely and affect yield considerably. Elahmadi (1994) reported that heat stress is one of the main constraints of wheat production and is known to cause stunted plant growth, reduced tillering and accelerated development leading to small heads, shriveled grains and low yield.

\section{Materials and Methods}

The experimental material for the present study consisted ten diverse wheat varieties (selected on the basis of genetic diversity, thermal tolerance and their stability for different yield traits), their all possible F1's (Excluding reciprocals) and F2's. The detail of each parent is given in Table 1.

Crosses among the ten genotypes were made in diallel fashion excluding reciprocals, during Rabi 2015-16 at RARI, Durgapura. A number of plants randomly selected in a parent were crossed with a number of randomly selected plants from other parent. In Karif 2016 half of the F1's seed was raised at Indian Agricultural Research Institute, Regional station, wallington (T.N.) to advance the generation. In Rabi 2016-17 ten genotypes along with their 45 F1's and 45 F2's progenies were evaluated in 2 environments (two different dates of sowing $15 \mathrm{Nov}$. and $05 \mathrm{Dec}$.) timely sown and late sown, with 3 replications at Agricultural Research Farm of Rajasthan Agricultural Research Institute (RARI)
Durgapura. Each replication contained two parts. First part consisted 10 parents and 45 F1's sown in two rows plot while the plots of second part consisted four rows of 45 F2's. Row length was 3 meter. Row to row and plant to plant distance was kept $30 \mathrm{~cm}$ and 10 $\mathrm{cm}$, respectively.

Observations were recorded for days to heading, days to maturity, plant height, flag leaf area, number of tillers per plant, spike length, number of grains per ear, biomass per plant, 1000-grain weight, harvest index and grain yield per plant, canopy temperature and protein content on 10 randomly selected plants in each of the F1's progenies along with each parent while 20 plants were selected in F2's population from each replication. Mean values over selected plants was used for analysis of heat susceptibility index.

Heat susceptibility index (HSI) was calculated for grain yield and other attributes over high temperature stress (late sown) and non-stress environment (normal sown) by using the formula as suggested by Fischer and Maurer (1978).

$\mathrm{HSI}=[1-\mathrm{YD} / \mathrm{YP}] / \mathrm{D}$

Where,

$\mathrm{YD}=$ mean of the genotypes in stress environment.

$\mathrm{YP}=$ mean of the genotypes under non-stress environment.

$\mathrm{D}=1-[$ mean $\mathrm{YD}$ of all genotypes/mean YP of all genotypes].

The HSI values were used to characterize the relative tolerance of genotypes based on minimization of yield losses compared to normal environmental conditions. The differences between genotypes for different characters were tested for significance by using standard techniques for analysis of variances. 


\section{Results and Discussion}

The impact of climate change is clearly evident from recent vagaries across regions in India. Although North-West Plain Zone (NWPZ) has weather conditions more favorable in comparison to Central Zone (CZ), the wheat yield under late sown condition is comparatively lower in this wheat bowl of the country (Mohan et. al., 2015). Hence, national programme leaders identified improving thermal tolerance of wheat as being a major research priority. Therefore, breeding aimed at selecting genotypes with thermal stress tolerance is one of the most vital objectives of the wheat breeder.

The results of present investigation demonstrated that the mean performance of parents, F1's and F2's under E2 (late sown) environment in comparison to E1 environment (normal sown). The results are in agreement with Ved Prakash (2007), Singh et. al. (2011), Tammam and Abd el-rady (2011) and Shashi Bala et. al. (2014). The heat susceptibility index (HSI) was calculated individually for each character in stress environment i.e. E2 (late sown) against E1 (Normal sown). On the basis of HSI, the parents, F1's and F2's were classified as highly tolerant, tolerant, moderately tolerant, and susceptible to heat stress.

Perusal of Table 2 indicated that parent Raj 1482, Raj 4120 and PBW 396 for days to heading; PBW 396, Raj 4083 and Raj 4120 for days to maturity; DBW 88, PBW 343 and Raj 4120 for plant height; DBW 88, Raj 1482 and PBW 396 for number of tillers per plant; PBW 502, Raj 4079 and DBW 88 for flag leaf area; Raj 4120,Raj 4083 and PBW 343 for spike length; Raj 4120, C 306 and PBW 343 for number of grains per spike; PBW 396,PBW 502 and Raj 4079 for 1000-grain weight; DBW 88,Raj 4083 and Raj 4079 for biological yield per plant; PBW 590, PBW 343 and Raj
1482 for harvest index; PBW 590, Raj 1482 and PBW 343 for canopy temperature; Raj 4120, PBW 590 and C 306 for protein content and PBW 343, Raj 4079, Raj 4083 and PBW 590 for grain yield per plant were least affected under late sown conditions (E2). An overall assessment of parents for all the studied characters indicated that PBW 343, Raj 4079, Raj 4083 and PBW 590 were found to be desirable for most of the characters on the basis of HSI.

Considering heat susceptibility index (HSI) in F1's (Table 2), the crosses PBW 343 x C 306, PBW 502 x Raj 4083, PBW 502 x Raj 4079, C 306 x Raj 4083 and PBW 396 x PBW 343 for days to heading; PBW $396 \times$ PBW 590, PBW 502 x PBW 396, PBW 396 x Raj 4120, PBW 502 x Raj 4079 and PBW 502 x PBW 590 for days to maturity; PBW 343 x C 306, PBW 396 x Raj 1482, C 306 x Raj 1482, Raj 1482 x Raj 4120 and C $306 \times$ Raj 4120 for plant height; Raj 1482 x Raj 4083, Raj 1482 x Raj 4079, PBW 343 x Raj 1482,PBW 502 x Raj 1482 and Raj 1482 x DBW 88 for number of tillers per plant; PBW 502 x PBW 343, PBW 343 x Raj 4079,PBW 502 x PBW 590, PBW 502 x Raj 4079 and PBW 502 x C 306 for flag leaf area; Raj 1482 x DBW 88, C 306 x PBW 590, Raj 1482 x Raj 4120, Raj 1482 x PBW 590 and PBW $502 \times$ Raj 4079 for spike length; Raj 4079 x DBW 88, PBW 590 x Raj 4120, PBW 343 x Raj 4120, PBW 396 x Raj 4120 and PBW $502 \times$ PBW 343 for number of grains per spike; PBW 396 x Raj 1482, PBW 396 x Raj 4079, PBW 502 x PBW 590, PBW 502 x Raj 4079 and PBW 502 x PBW 396 for 1000grain weight; C $306 \times$ PBW 590, PBW $502 \times$ PBW 343, PBW 343 x Raj 1482, Raj 4120 x Raj 4079 and PBW 396 x DBW 88 for biological yield per plant; Raj 4079 x DBW 88, PBW 502 x DBW 88, Raj 4083 x DBW 88, PBW 343 x Raj 4079 and PBW 502 x PBW 590 for harvest index; PBW $590 \times$ Raj 4079, PBW 502 x PBW 396, PBW 502 x C 306, PBW 502 x PBW 590 and Raj 1482 x 
Raj 4083 for canopy temperature; C 306 x Raj 4120,PBW 396 x Raj 1482, PBW 343 x C 306, PBW 396 x PBW 590 and PBW 590 x DBW 88 for protein content and PBW $343 \mathrm{x}$ Raj 4079, PBW 396 x Raj 4120,PBW 502 x PBW 590, C 306 x DBW 88 and PBW 502 x Raj 4079 for grain yield per plant exhibited comparatively more tolerance among $45 \mathrm{~F} 1$ 's under late sown conditions (E2). It is well established that grain yield is primary objective of any breeding programme, therefore F1 crosses PBW 343 x Raj 4079, PBW 396 x Raj 4120, PBW 502 x PBW 590, C 306 x DBW 88 and PBW 502 x Raj 4079 which revealed more tolerance for grain yield per plant were considered as desirable for thermal stress tolerance in E2.

Perusal of Table 2 revealed that in F2's, the crosses, PBW 343 x Raj 4079, PBW 590 x Raj 4079, PBW 590 x Raj 4083, Raj 4079 x Raj 4083 and PBW $343 \times$ Raj 4083 for days to heading; PBW 396 x PBW 590, PBW 343 x DBW 88,PBW 343 x PBW 590, PBW 502 x C 306 and PBW $502 \times$ PBW 396 for days to maturity; C 306 x Raj 4120, C 306 x PBW 590, PBW 396 x Raj 4079, Raj 1482 x DBW 88 and Raj 1482 x Raj 4083 for plant height; PBW 343 x Raj 4120, C 306 x Raj 4079, Raj 1482 x Raj 4079, Raj 4079 x Raj 4083 and Raj
$4120 \times$ DBW 88 for number of tillers per plant; PBW 343 x Raj 4079, Raj 4079 x Raj 4083, PBW 502 x DBW 88, PBW 590 x Raj 4079 and Raj 1482 x Raj 4079 for flag leaf area; PBW 502 x Raj 4083, C 306 x Raj 1482, PBW 502 x Raj 1482, PBW 502 x DBW 88 and PBW 502 x Raj 4120 for spike length; PBW 396 x Raj 4079, PBW 396 x PBW 590, PBW 343 x DBW 88, Raj 1482 x Raj 4079 and PBW $396 \times$ Raj 4120 for number of grains per spike; C 306 x Raj 4083, PBW 502 x Raj 4079, Raj 4120 x Raj 4083, PBW 343 x Raj 4120 and PBW $396 \times$ Raj 4079 for 1000-grain weight; Raj 4120 x DBW 88, PBW 343 x Raj 4079, PBW 590 x Raj 4083, PBW 343 x PBW 590 and Raj 4079 x Raj 4083 for biological yield per plant; PBW 502 x DBW 88, PBW 590 x DBW 88, PBW 502 x Raj 4079, C 306 $x$ DBW 88 and C 306 x Raj 1482 for harvest index; Raj 4079 x Raj 4083, PBW 590 x Raj 4079, Raj 1482 x DBW 88, PBW 502 x C 306 and PBW $590 \times$ Raj 4083 for canopy temperature; Raj 4120 x Raj 4083, C 306 x Raj 4120, PBW 590 x Raj 4120, Raj 1482 x DBW 88 and PBW $502 \times$ DBW 88 for protein content and PBW 502 x DBW 88, PBW 343 x Raj 4079, Raj 1482 x Raj 4120, PBW 343 x PBW 590 and Raj 4120 x Raj 4083 for grain yield per plant showed high heat tolerance under late sown conditions (E2).

Table.1 Pedigree of parental material of wheat used in the study

\begin{tabular}{|l|c|c|c|}
\hline S.N & Name of variety & Source & Pedigree \\
\hline $\mathbf{1 .}$ & PBW-502 & PAU, LUDHIANA & W485/PBW343//RAJ1482 \\
\hline $\mathbf{2 .}$ & PBW-396 & PAU, LUDHIANA & $\begin{array}{r}\text { CIANO-67/MARIS-FUNDIN //MONCHO/3/SERI- } \\
\text { 82; BARBET-1-SELECTION }\end{array}$ \\
\hline $\mathbf{3 .}$ & PBW-343 & PAU, LUDHIANA & ND /VG 1944 // KAL / BB / 3// YACO 's' / / / VEE \# 5 's' \\
\hline $\mathbf{4 .}$ & C-306 & CCSHAU, HISAR & RGN/CSK3//2*C591/3/C217/N14//C281 \\
\hline $\mathbf{5 .}$ & Raj-1482 & RARI, DURGAPURA & $\begin{array}{c}\text { NAPO63/TOBARI66//KALYANSONA/BLUEBIRD;CARTHA } \\
\text { GE//KALYANSONA/BLUEBIRD; }\end{array}$ \\
\hline $\mathbf{6 .}$ & PBW-590 & PAU, LUDHIANA & APO63/TOBARI66//II8156/3/KALYANSONA /BLUEBIRD \\
\hline $\mathbf{7 .}$ & Raj 4120 & RARI, DURGAPURA & WH594/RAJ3814//W485 \\
\hline $\mathbf{8 .}$ & Raj 4079 & RARI, DURGAPURA & PBW 373 / V1 \\
\hline $\mathbf{9 .}$ & Raj 4083 & RARI, DURGAPURA & UP2338/WH595 \\
\hline $\mathbf{1 0 .}$ & DBW -88 & IIWBR, KARNAL & PBW343/UP2442//WR258UP 2425 \\
\hline & & & KAUZ//ALTAR84/AOS/3/MILAN/ \\
\hline
\end{tabular}


Table.3 Heat susceptibility indices for yield and its contributing attributes in $E_{2}$ in comparison to $E_{1}$ environment

\begin{tabular}{|c|c|c|c|c|c|c|c|c|c|c|c|c|c|}
\hline Parents & $\begin{array}{l}\text { Days to } \\
\text { heading }\end{array}$ & $\begin{array}{l}\text { Days to } \\
\text { maturity }\end{array}$ & $\begin{array}{l}\text { Plant } \\
\text { height }\end{array}$ & $\begin{array}{l}\text { Number } \\
\text { of tillers } \\
\text { per plant }\end{array}$ & $\begin{array}{l}\text { Flag } \\
\text { leaf } \\
\text { area }\end{array}$ & $\begin{array}{l}\text { Spike } \\
\text { length }\end{array}$ & $\begin{array}{c}\text { Number } \\
\text { of grains } \\
\text { per ear }\end{array}$ & $\begin{array}{l}\text { 1000- } \\
\text { grain } \\
\text { weight }\end{array}$ & $\begin{array}{l}\text { Biomass } \\
\text { per plant }\end{array}$ & $\begin{array}{l}\text { Harvest } \\
\text { Index }\end{array}$ & $\begin{array}{l}\text { Grain yield } \\
\text { Per plant }\end{array}$ & $\begin{array}{l}\text { Canopy } \\
\text { temperature }\end{array}$ & Protein content \\
\hline PBW $502(\mathrm{P} 1)$ & 1.11 & 1.07 & 0.94 & 1.12 & 0.58 & 1.80 & 1.38 & 0.85 & 1.01 & 1.32 & 1.22 & 1.01 & 1.29 \\
\hline PBW 396 (P2) & 0.92 & 0.70 & 1.17 & 0.98 & 1.20 & 0.94 & 1.38 & 0.59 & 0.92 & 1.05 & 1.02 & 0.99 & 1.60 \\
\hline PBW 343 (P3) & 1.01 & 1.00 & 0.72 & 1.02 & 0.97 & 0.80 & 0.72 & 1.09 & 1.04 & 0.75 & 0.84 & 0.84 & 1.67 \\
\hline C 306 (P4) & 1.13 & 1.21 & 1.19 & 1.21 & 0.79 & 0.86 & 0.65 & 1.19 & 0.95 & 1.02 & 1.00 & 0.86 & 0.34 \\
\hline Raj 1482 (P5) & 0.47 & 1.08 & 1.34 & 0.75 & 1.45 & 1.09 & 1.16 & 1.18 & 1.43 & 0.77 & 0.96 & 0.59 & 1.46 \\
\hline PBW 590 (P6) & 1.19 & 0.97 & 0.95 & 1.09 & 1.33 & 1.79 & 1.10 & 0.92 & 1.55 & 0.62 & 0.90 & 0.54 & 0.32 \\
\hline Raj 4120 (P7) & 0.88 & 0.92 & 0.93 & 1.17 & 1.04 & 0.25 & 0.41 & 1.33 & 1.46 & 1.27 & 1.27 & 1.04 & -0.21 \\
\hline Raj 4079 (P8) & 1.17 & 1.11 & 1.02 & 1.27 & 0.59 & 0.94 & 0.91 & 0.89 & 0.71 & 0.92 & 0.87 & 1.15 & 1.24 \\
\hline Raj 4083 (P9) & 1.20 & 0.91 & 0.94 & 0.99 & 1.28 & 0.58 & 0.84 & 0.98 & 0.72 & 0.93 & 0.88 & 2.06 & 1.37 \\
\hline DBW 88 (P10) & 0.96 & 1.00 & 0.69 & 0.28 & 0.67 & 0.88 & 1.33 & 0.97 & 0.18 & 1.40 & 1.10 & 0.91 & 0.75 \\
\hline \multicolumn{14}{|l|}{$\mathrm{F}_{1}$ crosses } \\
\hline P1xP2 & 0.72 & 0.76 & 1.23 & 1.14 & 0.96 & 1.74 & 1.12 & 0.75 & 0.63 & 1.57 & 1.24 & -0.12 & 2.48 \\
\hline P1xP3 & 1.50 & 0.91 & 0.78 & 0.79 & 0.60 & 0.37 & 0.60 & 1.05 & -0.21 & 1.65 & 1.05 & 0.74 & 0.78 \\
\hline P1xP4 & 0.81 & 1.36 & 0.70 & 0.64 & 0.68 & 1.32 & 0.91 & 0.88 & 1.47 & 0.84 & 1.05 & 0.29 & 0.84 \\
\hline P1xP5 & 1.26 & 1.34 & 1.16 & 0.14 & 0.85 & 0.47 & 1.04 & 0.98 & 1.24 & 1.36 & 1.26 & 0.84 & 0.48 \\
\hline P1xP6 & 1.37 & 0.89 & 1.16 & 1.28 & 0.65 & 1.36 & 0.97 & 0.69 & 1.41 & 0.29 & 0.75 & 0.37 & 1.50 \\
\hline P1xP7 & 0.87 & 0.97 & 1.08 & 1.55 & 1.28 & 0.41 & 0.75 & 1.01 & 1.15 & 0.69 & 0.86 & 1.38 & 0.18 \\
\hline P1xP8 & 0.59 & 0.88 & 1.05 & 1.16 & 0.67 & 0.19 & 1.00 & 0.69 & 0.80 & 0.80 & 0.80 & 1.72 & 0.35 \\
\hline P1xP9 & 0.34 & 1.00 & 0.72 & 0.57 & 0.90 & 0.25 & 0.97 & 0.79 & 0.57 & 1.02 & 0.86 & 1.47 & 1.60 \\
\hline P1xP10 & 0.85 & 0.97 & 1.19 & 1.34 & 0.68 & 0.62 & 1.05 & 1.32 & 2.04 & 0.17 & 0.93 & 1.35 & 1.34 \\
\hline P2xP3 & 0.65 & 0.96 & 1.26 & 1.03 & 0.99 & 1.51 & 1.01 & 1.40 & 2.14 & 0.31 & 1.04 & 0.86 & 0.50 \\
\hline P2xP4 & 1.06 & 0.98 & 1.21 & 1.62 & 1.04 & 0.92 & 0.95 & 0.79 & 0.78 & 1.13 & 1.00 & 1.17 & 0.83 \\
\hline P2xP5 & 1.45 & 0.99 & 0.36 & 0.86 & 0.89 & 1.66 & 0.84 & 0.53 & 0.12 & 1.75 & 1.20 & 1.25 & -0.06 \\
\hline P2xP6 & 1.55 & 0.73 & 0.82 & 0.83 & 0.96 & 1.70 & 0.70 & 0.75 & 1.85 & 0.94 & 1.23 & 1.52 & 0.06 \\
\hline P2xP7 & 0.94 & 0.86 & 1.12 & 0.61 & 0.90 & 0.38 & 0.49 & 0.88 & 0.71 & 0.75 & 0.75 & 1.73 & 1.00 \\
\hline P2xP8 & 0.97 & 0.90 & 0.86 & 1.52 & 0.82 & 1.56 & 1.03 & 0.63 & 1.41 & 0.58 & 0.89 & 1.42 & 0.66 \\
\hline P2xP9 & 0.88 & 0.91 & 0.94 & 0.57 & 1.07 & 0.36 & 1.51 & 1.00 & 1.41 & 0.89 & 1.07 & 1.09 & 1.06 \\
\hline P2xP10 & 0.97 & 0.90 & 1.16 & 1.33 & 1.22 & 0.38 & 1.07 & 0.98 & 0.03 & 1.54 & 1.04 & 0.47 & 1.11 \\
\hline P3xP4 & 0.20 & 0.97 & 0.36 & 1.40 & 0.87 & 0.30 & 1.53 & 0.98 & 0.89 & 1.23 & 1.09 & 1.30 & -0.06 \\
\hline P3xP5 & 0.88 & 0.98 & 1.01 & 0.10 & 0.95 & 1.70 & 1.07 & 1.00 & -0.06 & 1.56 & 1.02 & 0.81 & 1.00 \\
\hline P3xP6 & 1.07 & 0.95 & 1.06 & 1.55 & 1.22 & 2.20 & 1.10 & 1.32 & 1.06 & 0.74 & 0.86 & 1.02 & 0.84 \\
\hline P3xP7 & 1.09 & 1.22 & 1.15 & 0.53 & 1.15 & 1.22 & 0.47 & 1.35 & 0.90 & 1.53 & 1.27 & 1.24 & 0.60 \\
\hline P3xP8 & 0.80 & 1.19 & 1.44 & 1.33 & 0.61 & 0.94 & 0.93 & 1.06 & 0.99 & 0.23 & 0.55 & 1.33 & 1.44 \\
\hline P3xP9 & 0.78 & 1.19 & 1.64 & 1.86 & 1.76 & 1.63 & 0.74 & 1.02 & 0.89 & 1.12 & 1.03 & 1.01 & 0.96 \\
\hline P3xP10 & 1.55 & 0.93 & 1.04 & 1.42 & 1.05 & 1.60 & 0.78 & 1.25 & 1.26 & 1.02 & 1.08 & 0.80 & 1.57 \\
\hline
\end{tabular}




\begin{tabular}{|c|c|c|c|c|c|c|c|c|c|c|c|c|c|}
\hline P4xP5 & 0.81 & 1.00 & 0.48 & 0.81 & 1.24 & 0.33 & 1.08 & 0.95 & 0.71 & 1.25 & 1.04 & 0.83 & 0.55 \\
\hline P4xP6 & 1.29 & 0.96 & 0.89 & 1.91 & 1.11 & 0.03 & 0.99 & 1.00 & -0.54 & 2.12 & 1.31 & 1.05 & 1.17 \\
\hline P4xP7 & 0.95 & 0.94 & 0.53 & 0.92 & 0.97 & 0.45 & 0.98 & 1.15 & 1.85 & 0.57 & 1.05 & 0.76 & -4.33 \\
\hline P4xP8 & 0.74 & 0.93 & 0.96 & 0.32 & 1.18 & 1.46 & 1.01 & 1.16 & 1.76 & 0.63 & 1.06 & 0.95 & 0.77 \\
\hline P4xP9 & 0.61 & 0.98 & 0.86 & 0.27 & 1.14 & 0.93 & 1.16 & 1.04 & 1.81 & 0.33 & 0.92 & 0.82 & 0.65 \\
\hline P4xP10 & 1.12 & 0.94 & 1.24 & 1.50 & 0.99 & 1.10 & 0.77 & 1.09 & 0.88 & 0.65 & 0.75 & 1.74 & 2.65 \\
\hline P5xP6 & 0.88 & 0.96 & 0.56 & 0.89 & 0.94 & 0.03 & 1.42 & 0.96 & 1.05 & 0.94 & 0.97 & 0.72 & 1.88 \\
\hline P5xP7 & 1.06 & 1.10 & 0.50 & 0.71 & 1.04 & 0.03 & 1.57 & 0.92 & 1.29 & 0.95 & 1.05 & 1.05 & 2.11 \\
\hline P5xP8 & 0.94 & 0.96 & 0.67 & -0.13 & 0.95 & 1.41 & 1.17 & 1.01 & 1.33 & 0.75 & 0.96 & 0.80 & 2.46 \\
\hline P5xP9 & 0.93 & 0.97 & 0.97 & -0.41 & 1.24 & 0.97 & 1.30 & 1.06 & 1.46 & 0.70 & 0.98 & 0.40 & 0.96 \\
\hline P5xP10 & 0.88 & 0.96 & 0.60 & 0.15 & 1.04 & -0.25 & 1.23 & 0.97 & 1.20 & 0.60 & 0.84 & 0.62 & 1.21 \\
\hline P6xP7 & 1.55 & 0.99 & 1.11 & 1.27 & 0.95 & 0.32 & 0.27 & 0.82 & 1.60 & 0.63 & 0.99 & 0.67 & 0.49 \\
\hline P6xP8 & 1.55 & 0.98 & 1.18 & 1.37 & 0.76 & 2.29 & 0.94 & 1.05 & 0.77 & 1.84 & 1.42 & -0.17 & 2.28 \\
\hline P6xP9 & 0.92 & 1.17 & 1.26 & 1.08 & 1.28 & 1.45 & 1.09 & 1.41 & 0.60 & 1.56 & 1.20 & 0.96 & 2.86 \\
\hline P6xP10 & 0.87 & 1.02 & 1.20 & 1.86 & 0.87 & 0.74 & 1.32 & 0.96 & 0.04 & 1.42 & 0.96 & 0.45 & 0.12 \\
\hline P7xP8 & 1.21 & 1.16 & 1.19 & 0.90 & 0.93 & 0.24 & 1.07 & 0.82 & -0.03 & 1.40 & 0.93 & 1.43 & 0.36 \\
\hline P7xP9 & 1.23 & 0.98 & 1.13 & 1.30 & 0.97 & 0.31 & 1.59 & 0.81 & 0.59 & 1.16 & 0.96 & 1.34 & 0.31 \\
\hline P7xP10 & 1.00 & 0.93 & 1.16 & 1.16 & 1.18 & 0.35 & 1.15 & 0.88 & 0.40 & 1.04 & 0.82 & 1.76 & 1.18 \\
\hline P8xP9 & 0.95 & 0.97 & 1.61 & 1.22 & 0.87 & 2.26 & 0.71 & 1.75 & 0.48 & 1.02 & 0.84 & 1.14 & 1.63 \\
\hline P8xP10 & 1.01 & 1.02 & 1.10 & 0.41 & 0.94 & 2.31 & 0.23 & 0.95 & 1.85 & 0.13 & 0.83 & 1.22 & 1.30 \\
\hline P9xP10 & 1.08 & 1.28 & 1.13 & 0.86 & 1.31 & 1.00 & 0.96 & 0.77 & 1.81 & 0.21 & 0.86 & 1.05 & 1.39 \\
\hline \multicolumn{14}{|c|}{$\mathrm{F}_{2}$ crosses } \\
\hline P1xP2 & 0.55 & 0.74 & 0.84 & 0.44 & 1.06 & 2.22 & 1.48 & 1.16 & 0.65 & 1.63 & 1.11 & 0.74 & 1.62 \\
\hline P1xP3 & 1.15 & 1.23 & 0.73 & 0.26 & 0.98 & 0.96 & 0.97 & 1.00 & 0.92 & 0.89 & 0.91 & 1.03 & 0.56 \\
\hline P1xP4 & 1.09 & 0.71 & 1.00 & 1.04 & 0.87 & 1.18 & 0.35 & 1.07 & 1.39 & 0.87 & 1.14 & 0.10 & 1.00 \\
\hline P1xP5 & 1.48 & 0.96 & 1.22 & 1.37 & 0.90 & 0.21 & 1.29 & 0.99 & 0.53 & 2.03 & 1.24 & 0.68 & 1.17 \\
\hline P1xP6 & 0.73 & 1.12 & 1.24 & 1.65 & 1.04 & 1.13 & 0.59 & 0.77 & 0.61 & 0.97 & 0.79 & 0.38 & 0.45 \\
\hline P1xP7 & 1.41 & 0.84 & 1.18 & 0.80 & 0.92 & 0.42 & 0.63 & 1.48 & 0.94 & 1.78 & 1.30 & 0.34 & 0.25 \\
\hline P1xP8 & 1.17 & 1.01 & 1.12 & 1.35 & 1.19 & 0.72 & 1.26 & 0.58 & 1.72 & 0.14 & 1.03 & 1.33 & 0.50 \\
\hline P1xP9 & 0.64 & 0.94 & 0.64 & 0.37 & 1.37 & -0.05 & 0.78 & 0.90 & 1.14 & 0.23 & 0.74 & 2.13 & 0.89 \\
\hline P1xP10 & 0.49 & 1.18 & 1.25 & 1.60 & 0.76 & 0.32 & 0.64 & 1.35 & 0.80 & 0.04 & 0.48 & 1.29 & 0.25 \\
\hline P2xP3 & 1.52 & 1.04 & 1.20 & 0.73 & 0.90 & 1.02 & 1.94 & 0.98 & 1.32 & 0.89 & 1.11 & 1.10 & 1.63 \\
\hline P2xP4 & 0.50 & 1.26 & 1.17 & 0.40 & 1.02 & 0.89 & 1.71 & 0.89 & 0.96 & 0.81 & 0.90 & 1.79 & 1.63 \\
\hline P2xP5 & 1.09 & 0.89 & 0.65 & 0.74 & 1.08 & 1.15 & 0.31 & 0.97 & 0.87 & 1.02 & 0.94 & 0.91 & 0.38 \\
\hline P2xP6 & 1.28 & 0.59 & 0.80 & 1.12 & 1.06 & 1.24 & 0.14 & 0.80 & 0.68 & 1.64 & 1.12 & 1.58 & 0.99 \\
\hline P2xP7 & 1.12 & 1.47 & 1.24 & 1.73 & 0.86 & 0.42 & 0.31 & 1.28 & 1.89 & 0.25 & 1.16 & 1.24 & 1.22 \\
\hline P2xP8 & 1.48 & 1.01 & 0.61 & 0.45 & 1.03 & 0.68 & -0.15 & 0.75 & 1.22 & 0.30 & 0.82 & 1.95 & 2.94 \\
\hline P2xP9 & 1.55 & 0.97 & 0.73 & 0.72 & 1.18 & 0.80 & 0.93 & 0.77 & 1.41 & 0.77 & 1.11 & 1.42 & 1.30 \\
\hline P2xP10 & 0.84 & 0.92 & 0.87 & 1.71 & 0.87 & 0.84 & 0.75 & 1.07 & 0.74 & 1.30 & 0.99 & 0.35 & 0.99 \\
\hline P3xP4 & 1.08 & 0.74 & 0.72 & 2.09 & 0.98 & 1.03 & 1.95 & 1.08 & 1.25 & 1.07 & 1.14 & 0.92 & 1.18 \\
\hline P3xP5 & 1.32 & 0.83 & 1.22 & 0.82 & 1.21 & 1.01 & 1.12 & 1.28 & 1.31 & 1.00 & 1.14 & 1.41 & 0.57 \\
\hline
\end{tabular}




\begin{tabular}{|c|c|c|c|c|c|c|c|c|c|c|c|c|c|}
\hline P3xP6 & 0.79 & 0.70 & 0.75 & 0.27 & 1.00 & 1.03 & 1.17 & 1.09 & 0.36 & 0.99 & 0.68 & 0.60 & 2.32 \\
\hline P3xP7 & 1.20 & 1.27 & 0.64 & -0.03 & 0.90 & 1.02 & 1.04 & 0.67 & 0.56 & 2.06 & 1.28 & 0.34 & 1.63 \\
\hline P3xP8 & -0.76 & 1.32 & 0.99 & 1.04 & 0.49 & 0.98 & 0.98 & 0.95 & 0.33 & 0.91 & 0.62 & 1.40 & 2.52 \\
\hline P3xP9 & 0.10 & 1.49 & 0.72 & 1.93 & 1.30 & 0.98 & 0.83 & 0.84 & 1.31 & 0.58 & 0.97 & 1.34 & 4.25 \\
\hline P3xP10 & 1.63 & 0.70 & 0.92 & 1.61 & 1.36 & 1.77 & 0.24 & 0.90 & 0.67 & 1.52 & 1.06 & 1.39 & 0.50 \\
\hline P4xP5 & 1.05 & 0.92 & 0.72 & 1.39 & 1.07 & 0.00 & 1.23 & 0.84 & 1.25 & 0.17 & 0.79 & 1.50 & 1.90 \\
\hline P4xP6 & 0.64 & 0.98 & 0.58 & 1.79 & 0.89 & 0.97 & 0.82 & 1.77 & 0.78 & 1.02 & 0.90 & 1.77 & 0.57 \\
\hline P4xP7 & 1.39 & 0.96 & 0.58 & 1.01 & 1.14 & 1.17 & 0.85 & 0.90 & 0.76 & 0.79 & 0.80 & 2.01 & 0.13 \\
\hline P4xP8 & 1.09 & 0.99 & 1.22 & 0.00 & 0.94 & 1.11 & 1.56 & 1.04 & 1.73 & 0.75 & 1.26 & 0.53 & 0.63 \\
\hline P4xP9 & 0.90 & 1.02 & 1.58 & 1.16 & 1.18 & 1.89 & 1.01 & 0.13 & 1.14 & 1.29 & 1.18 & 1.04 & 0.38 \\
\hline P4xP10 & 1.12 & 1.22 & 1.27 & 1.45 & 0.99 & 1.56 & 0.39 & 0.75 & 1.81 & 0.15 & 1.09 & 0.83 & 0.31 \\
\hline P5xP6 & 1.49 & 0.98 & 1.09 & 0.89 & 0.81 & 0.75 & 1.57 & 1.00 & 0.73 & 1.46 & 1.07 & 1.31 & 1.12 \\
\hline P5xP7 & 1.51 & 0.79 & 1.15 & 0.60 & 0.91 & 0.78 & 1.85 & 0.98 & 0.62 & 0.65 & 0.65 & 0.74 & 1.06 \\
\hline P5xP8 & 1.40 & 1.00 & 1.20 & 0.00 & 0.77 & 0.63 & 0.23 & 1.15 & 0.77 & 1.69 & 1.19 & 1.06 & 0.63 \\
\hline P5xP9 & 0.90 & 1.04 & 0.62 & 1.08 & 0.97 & 1.30 & 1.21 & 0.97 & 1.58 & 1.03 & 1.29 & 0.56 & 1.06 \\
\hline P5xP10 & 1.12 & 0.98 & 0.61 & 2.21 & 1.00 & 0.60 & 0.79 & 0.78 & 1.05 & 1.61 & 1.27 & 0.00 & 0.19 \\
\hline P6xP7 & 0.64 & 1.04 & 1.19 & 1.46 & 0.96 & 0.87 & 1.39 & 0.87 & 1.00 & 1.67 & 1.28 & 0.56 & 0.19 \\
\hline P6xP8 & -0.05 & 0.91 & 0.78 & 0.45 & 0.76 & 1.22 & 0.93 & 0.87 & 0.47 & 1.11 & 0.79 & -0.15 & 0.51 \\
\hline P6xP9 & 0.05 & 0.90 & 0.99 & 1.27 & 1.01 & 1.06 & 0.58 & 1.54 & 0.35 & 1.04 & 0.70 & 0.12 & 0.62 \\
\hline P6xP10 & 1.41 & 0.98 & 1.18 & 0.71 & 1.05 & 1.18 & 1.66 & 0.98 & 1.31 & 0.11 & 0.79 & 0.97 & 0.81 \\
\hline P7xP8 & 1.40 & 1.11 & 1.21 & 0.56 & 1.00 & 1.55 & 1.03 & 0.81 & 1.16 & 0.28 & 0.78 & 2.04 & 0.06 \\
\hline P7xP9 & 1.36 & 1.10 & 1.22 & 1.58 & 0.96 & 1.14 & 1.19 & 0.60 & 0.90 & 0.42 & 0.69 & 0.65 & 0.38 \\
\hline P7xP10 & 1.37 & 0.98 & 1.21 & 0.19 & 1.25 & 1.01 & 1.62 & 0.96 & 0.09 & 2.36 & 1.24 & 1.35 & 0.75 \\
\hline P8xP9 & 0.09 & 1.00 & 1.21 & 0.00 & 0.52 & 1.85 & 0.98 & 1.69 & 0.44 & 2.13 & 1.26 & -0.41 & 0.58 \\
\hline P8xP10 & 0.91 & 0.97 & 1.38 & 0.60 & 1.16 & 0.43 & 0.43 & 1.41 & 1.54 & 0.23 & 0.97 & 0.90 & 0.74 \\
\hline P9xP10 & 0.64 & 1.01 & 1.11 & 1.36 & 1.12 & 1.15 & 1.42 & 0.85 & 1.54 & 0.52 & 1.07 & 1.25 & 0.75 \\
\hline
\end{tabular}


On the basis of HSI for grain yield in F2 generation PBW 502 x DBW 88, PBW 343 x Raj 4079, Raj 1482 x Raj 4120, PBW 343 x PBW 590 and Raj 4120 x Raj 4083 were considered as desirable for thermal stress tolerance in E2. Resemblance across the generations indicated the superiority of the crosses PBW $502 \times$ PBW 590, PBW 502 x Raj 4083, PBW 502 x DBW 88, PBW 396 x Raj 4079, PBW 343 x PBW 590, PBW 343 x Raj 4079, PBW 590 x DBW 88, Raj 4120 x Raj 4079, Raj 4120 x Raj 4083 and Raj 4079 $\mathrm{x}$ DBW 88 under late sown condition (E2). Low value of heat stress intensity (D- value) indicated that parameters viz., days to heading, tillers per plant, spike length, 1000grain weight, biological yield, canopy temperature and protein content were less affected while days to maturity, plant height, flag leaf area, grains per spike, harvest index, grain yield per plant with high heat stress intensity (D- value) suffered more under E2 environment.

A close appraisal of Table 2 indicated that among the parents, PBW 343, Raj 4079, Raj 4083 and PBW 590; in F1 crosses PBW 343 x Raj 4079, PBW 396 x Raj 4120, PBW 502 x PBW 590, C 306 x DBW 88 and PBW 502 x Raj 4079 and in F2, PBW 502 x PBW 590, PBW 502 x Raj 4083, PBW 502 x DBW 88, PBW 396 x Raj 4079, PBW 343 x PBW 590, PBW 343 x Raj 4079, PBW 590 x DBW 88, Raj 4120 x Raj 4079, Raj 4120 x Raj 4083 and Raj 4079 x DBW 88 were least affected under late sown conditions (E2). Ved Prakash et. al. (2007) also reported higher D-value for grain yield $/ \mathrm{m} 2$.

On the basis of heat susceptibility index, the parents PBW 343, Raj 4079, Raj 4083 and PBW 590 were most desirable parents in E2. As a consequence, it is recommended that these genotypes may perform as potential donor for heat tolerance. These parents should be further exploited for improvement of grain yield under late and very late sown conditions. The HSI should be taken as important criteria for breeding wheat genotypes suitable for late sown conditions.

\section{References}

Anonymous, 2016. Food Outlook, Biannual Report on Global Food Markets, FAO Trade and Markets Division. ISSN: 0251-1959 (print) p. 12.

Anonymous, 2017. Progress report of All India Coordinated Wheat \& Barley Improvement Project 2016-17, Director's Report. Ed. G.P. Singh, ICAR-Indian Institute of Wheat \& Barley Research, Karnal, India.

Anonymous.2017. Directorate of agriculture, Rajsthan, Jaipur.

Elahmadi, A.B. (1994). Development of wheat germplasm tolerant to heat stress in Sudan. In: Saunders DA, Hettel GP (Eds.), Wheat in heat stressed environments: Irrigated, dry areas and rice-wheat farming systems. CIMMYT, Mexico DF.

Evans, L.T. 1993. Crop evolution, adaptation and yield. Cambridge University Press, Cambridge, U.K.

Fisher, R. A. and Maurer, R. 1978. Drought resistance in spring wheat cultivars I. Grain yield responses. Aust. J. Agric. Res., 29 (5):897-912.

Mohan, D., Mamrutha, H.M., Tyagi, B.S. and Sharma, Y. (2015). Weather conditions favouring high wheat productivity at some sites of madhya pradesh under late-sown condition. Wheat and Barley Newsletter, ICAR-IIWBR, Karnal, 9 (1 \& 2).

Shewry, P.R. 2009. Wheat. Journal of Experimental Botany, 60: 1537-1553.

Shashi Bala, Asthir, B. and Bains, N.S. 2014. Effect of terminal heat stress on yield and yield attributes of wheat. Int. J. App. Res., 4 (6): 1-2. 
Singh, K., Sharma, S. N. and Sharma, Y. 2011. Effect of high temperature on yield attributing traits in bread wheat. Bangladesh J. Agril. Res., 36 (3): 415426.

Tammam, A.M. and Abd el-rady, A.G. 2011. Genetical studies on some morphophysiological traits in some bread wheat crosses under heat stress conditions. Egypt. J. Agric. Res., 89 (2): 589-604.

Ved Praksh. 2007. Screening of wheat (Triticum aestivum L.) genotypes under limited moisture and heat stress environments. Indian J. Genet., 67 (1): 31- 33 .

\section{How to cite this article:}

Pawan Kumar, Hoshiyar Singh, Jhabar Singh and Choudhary, R.N. 2018. Estimation of Heat Stress Tolerance for Yield and its Contributing Attributes in Bread Wheat. Int.J.Curr.Microbiol.App.Sci. 7(07): 3817-3825. doi: https://doi.org/10.20546/ijcmas.2018.707.443 